\title{
APOBEC3G acts as a therapeutic target in mesenchymal gliomas by sensitizing cells to radiation-induced cell death
}

\author{
Yu Wang ${ }^{1,4, *}$, Shaofang Wu ${ }^{1, *}$, Siyuan Zheng ${ }^{2}$, Shuzhen Wang ${ }^{1}$, Arjun Wali ${ }^{1}$, \\ Ravesanker Ezhilarasan ${ }^{3}$, Erik P. Sulman ${ }^{3}$, Dimpy Koul ${ }^{1}$ and W.K. Alfred Yung ${ }^{1}$ \\ ${ }^{1}$ Brain Tumor Center, Departments of Neuro-Oncology, The University of Texas MD Anderson Cancer Center, Houston, Texas, \\ USA \\ ${ }^{2}$ Genomic Medicine, The University of Texas MD Anderson Cancer Center, Houston, Texas, USA \\ ${ }^{3}$ Radiation Oncology, The University of Texas MD Anderson Cancer Center, Houston, Texas, USA \\ ${ }^{4}$ Department of Neurosurgery, Peking Union Medical College Hospital, Chinese Academy of Medical Sciences and Peking \\ Union Medical College, Beijing, China \\ *These authors contributed equally to this work
}

Correspondence to: W.K. Alfred Yung, email: wyung@mdanderson.org

Dimpy Koul, email: dkoul@mdanderson.org

Keywords: mesenchymal gliomas, APOBEC3G, TGF $\beta$ signaling, radiation and cell death

Received: November 02, $2016 \quad$ Accepted: April 11, $2017 \quad$ Published: April 21, 2017

Copyright: Wang et al. This is an open-access article distributed under the terms of the Creative Commons Attribution License 3.0 (CC BY

3.0), which permits unrestricted use, distribution, and reproduction in any medium, provided the original author and source are credited.

\section{ABSTRACT}

Genomic, transcriptional, and proteomic analyses of brain tumors reveal that subtypes differ in their pathway activity, progression, and response to therapy. We performed an expression profiling of Glioma Initiating Cells (GICs) and comparative analysis between different groups of GICs indicates major variations in gene expression. Hierarchical clustering analysis revealed groups of GICs reflecting their heterogeneity, and among some of the genes as major regulators of mesenchymal phenotype, we identified $A B O B E C 3 G$ as one of the most discriminating genes in mesenchymal group. ABOBEC3G revealed a strong correlation with overall survival in TCGA GBM patient cohorts. APOBEC3G regulates cell invasion and silencing of this gene in GICs inhibits cell invasion and also glioma sphere initiation. APOBEC3G controls invasion through TGF $\beta /$ Smad 2 pathway by regulating Smad 2 target genes Thrombospondin 1, matrix metallopeptidase 2 and TIMP metallopeptidase inhibitor 1. We also show that targeting APOBEC3G can sensitize cancer cells to radiation induced cell death by attenuating activation of the DNA repair pathway. This response is mainly shown by decreased pChk2 expression in knockdown APOBEC3G cells. Taken together, we show that APOBEC3G gene is a mesenchymal enriched gene that controls invasion and knockdown of APOBEC3G sensitizes cells to radiation induced cell death, suggesting that APOBEC3G can be considered for use in stratifying patients with GBM for prognostic considerations.

\section{INTRODUCTION}

Glioblastoma (GBM), the most common primary malignant tumor of the central nervous system [1], is also most aggressive, with a dismal prognosis. The median survival time of patients with GBM is less than 2 years despite standard care, composed of resection, concomitant radiotherapy plus temozolomide, and adjuvant chemotherapy with temozolomide [2]. Few patients survive more than 5 years [3].
Although a great effort has been made to understand the molecular mechanism of GBM, no meaningful survival improvements have been resulted. Recent research has been focused on identifying new targets against GBM [4]. The Cancer Genome Atlas (TCGA) divided GBM into four subtypes: classic, mesenchymal, neural, and proneural [5]. Transcriptional profiling studies by Phillips et al. [6] and Verhaak et al. [5] have revealed molecular subtypes of high-grade gliomas based on the expression of genes characteristic of proneural $(\mathrm{PN})$, neural $(\mathrm{N})$, classical 
(CLAS) or mesenchymal (MES). A parallel comparison of these two studies revealed particularly strong agreement in the gene signatures associated with the PN and MES subtypes [7]. A number of transcription factors, including C/EBP- $\beta$ (CCAAT-enhancer-binding protein- $\beta$ ) and STAT3 (signal transducer and activator of transcription 3 ) and more recently the transcriptional coactivator TAZ (transcriptional coactivator with PDZ-binding motif), have been identified as important regulators of the mesenchymal phenotype in GBM $[8,9]$.

The APOBEC (apolipoprotein B mRNA editing catalytic polypeptide-like) family of proteins are a group of DNA-editing enzymes that play an important role in the innate immune response to retroviruses and retrotransposons [10]. APOBEC3G is a member of this family that can restrict HIV1 infection by viral strains that lack viral infectivity factor $[11,12]$. During reverse transcription in HIV-infected cells, the virion-packaged APOBEC3G deaminates cytidine residues to uridine in minus-strand DNA and causes guanosine-to-adenosine hypermutation in the opposite strand, resulting in inactivation of the viral genome [13-15]. APOBEC3G inhibits elongation of $\mathrm{HIV}-1$ reverse transcripts by inhibiting the translocation of reverse transcriptase along template RNA [16]. In addition, A3G sensitized cells to recognition by NK cells through upregulation of the expression of NKG2D ligands in HIV-infected cells to enhance the immune response and help the defense against pathogens [15]. Apart from contributing to immunity against viral infection, recent studies suggested APOBEC $3 \mathrm{G}$ also exerts important non-antiviral functions in cancer. APOBEC3G induces oncogenic transformation [17] and promotes liver metastasis and is correlated with poor prognosis in colon carcinoma patients with hepatic metastasis $[18,19]$. While APOBEC3A and APOBEC3B are directly linked to cancer through a DNA deaminasedependent mechanism [20], this is not the case for APOBEC3G as it is retained in cytoplasm [21]. Actually, the mechanism of APOBEC $3 \mathrm{G}$ in tumors, especially in GBM, is largely unknown.

Transforming growth factor- $\beta$ (TGF $\beta$ ) has a key role in tissue homeostasis and cancer, and elevated TGF $\beta$ activity has been associated with poor clinical outcome in high-grade glioma $[22,23]$. TGF $\beta$ can promote epithelialto-mesenchymal transition in epithelial cancers, leading to enhanced migration and invasion capacities in these cells $[24,25]$. It is conceivable that the mesenchymal subtype of GBM has similar mechanisms [26]. TGF $\beta$ is highly activated in the mesenchymal subtype of GBMs, but whether APOBEC $3 \mathrm{G}$ has crosstalk with TGF $\beta$ remains to be elucidated. APOBEC $3 \mathrm{G}$ has been shown to enhance lymphoma cell radioresistance by promoting DNA repair [27]. Since radiation therapy is one of the backbones of the treatment of GBM, whether APOBEC $3 \mathrm{G}$ can promote radioresistance in GBM is clinically significant. Irradiation (IR) mainly causes cell death through the induction of
DNA double-strand breaks (DSBs), which activates the DNA repair pathway. Checkpoint kinase 2 (Chek2, Chk2) is an important signal transducer of cellular responses to DNA damage. Activation of Chk2, especially when it is phosphorylated at threonine 68 , can initiate a multistep dynamic process to repair DNA damage [28, 29].

In this study, we screened GICs and TCGA data and found that APOBEC $3 \mathrm{G}$ was highly expressed in mesenchymal GBM; it was also associated with significantly decreased survival time in GBM patients, suggesting that it is a tumor-promoting factor in GBM. We identified APOBEC3G as a survivor factor of mesenchymal GBM after radiation. We show TGF $\beta$ signaling pathway regulated by APOBEC $3 \mathrm{G}$ that was associated with enhanced tumor invasion in GBM. Targeting APOBEC3G sensitizes GBM cell lines to IR by attenuating activation of the DNA repair pathway.

\section{RESULTS}

\section{APOBEC3G Is highly expressed in mesenchymal subtype of GICs and GBM cell lines}

Transcriptional analyses of brain tumors divide glioblastomas into different subtypes with characteristic pathway activity and response to therapy. Based on the transcriptome array analyses, we found that genes are differentially expressed between Mesenchymal GICs and other non-Mesenchymal GICs. We compared mesenchymal and non-mesenchymal GIC lines ( $n=7$ and $n=19$, respectively) using the significance analysis of microarrays [30]. Applying a FDR of $\leq 0.01$, we identified 89 genes significantly upregulated and 164 genes downregulated in mesenchymal GICs (Supplementary Dataset 1). Among the upregulated genes, APOBEC3G showed strong upregulation in the mesenchymal subtype (fold change $=8.94$ ) (Supplementary Table 1$)$. Of the 89 -upregulated genes, 87 were included in the TCGA U133 platform and 83 (95.4\%) also showed upregulation (fold change $>1$ ) in the mesenchymal subtype. Among the downregulated genes, 154 were included in TCGA U133A platform, 147 of which (95.4\%) showed downregulation. Among the upregulated genes, APOBEC3G also showed strong upregulation in the mesenchymal subtype (fold change $=1.84)($ Supplementary Dataset 2 , Supplementary Figure 1).

Analysis of expression of GICs (Figure 1A) and TCGA data (Figure 1B) revealed that APOBEC3G was enriched in mesenchymal subgroup of GBMs with CD44 as the mesenchymal marker and Olig-2 as a non-mesenchymal marker. We found the expression of APOBEC3G was correlated with CD44 (Spearman's correlation: 0.45) (Figure 1C). To validate the mRNA expression data, we used Western blot analysis to confirm the protein expression of APOBEC $3 \mathrm{G}$ in several GICs and GBM cell lines. Concordant with the 
TCGA patient microarray data, APOBEC3G protein was highly expressed in mesenchymal GBM cell lines and GICs but not in non- mesenchymal GICs (Figure 1D). Kaplan-Meier plots and log-rank survival analyses showed that the median overall survival time of the highAPOBEC3G group was markedly shorter than that of the low-APOBEC3G groups, suggesting that APOBEC3G is associated with poor clinical outcomes $(P<0.01)$ (Figure 1E).

\section{Targeting APOBEC3G attenuates proliferation of mesenchymal GICs and GBM cells}

To assess the functional significance of the relative overexpression of APOEC3G in $\mathrm{CD}_{4} 4^{+}$mesenchymal glioma cells compared with $\mathrm{CD}_{4} 4^{-}$glioma cells, we depleted APOBEC3G expression using lentivirus expressing shRNA directed against APOBEC3G in A172, U343, and GSC20 cells (Figure 2A). Knock-down

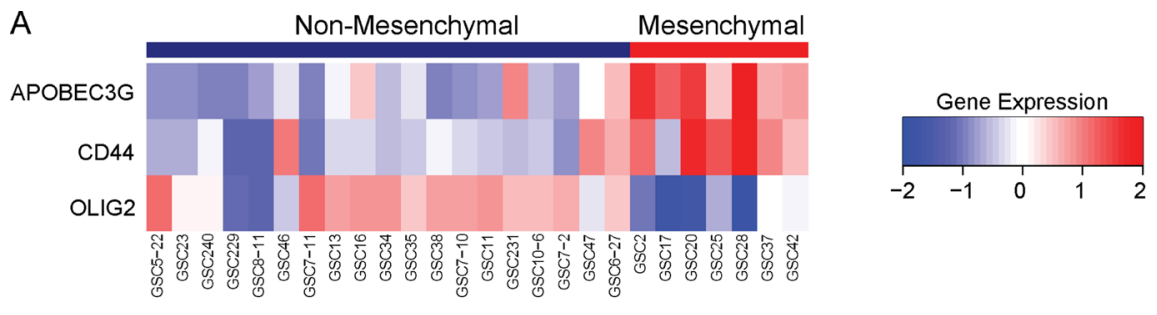

B

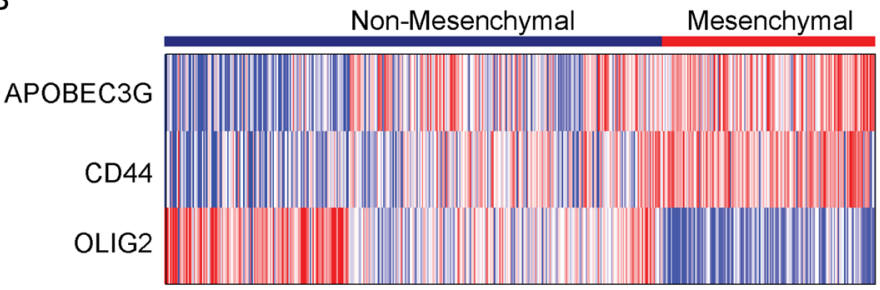

C

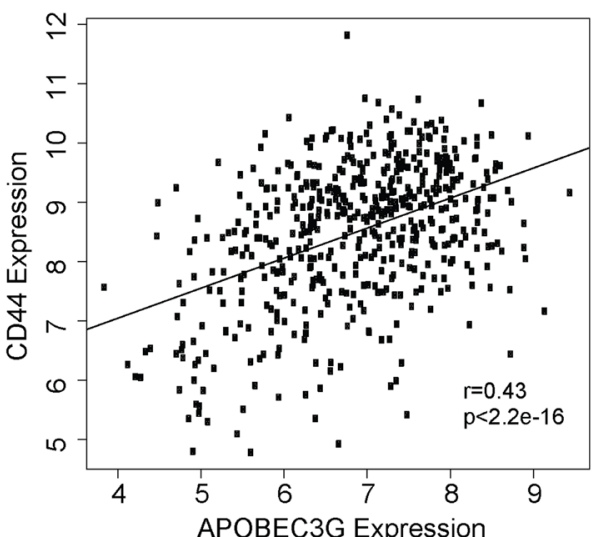

E

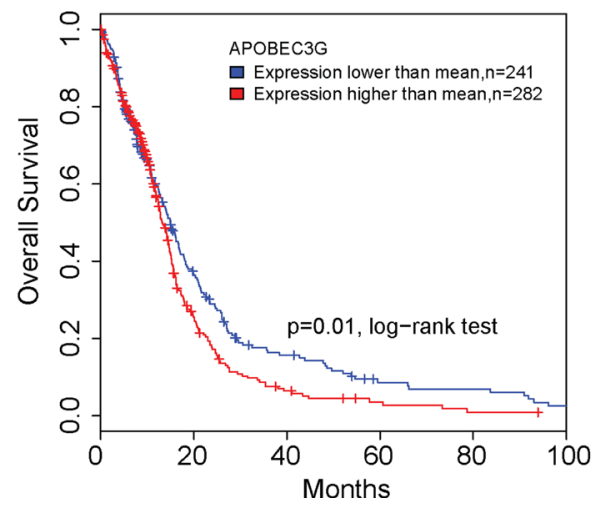

D

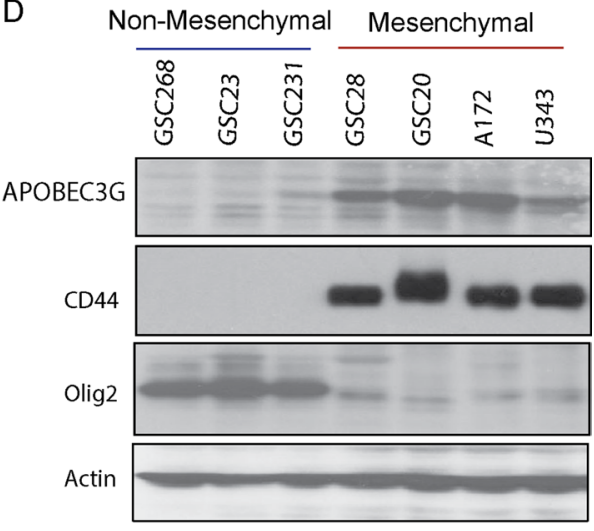

Figure 1: APOBEC3G was highly expressed in mesenchymal subtype of GICs and GBM cell lines. (A) Gene expression analysis of APOBEC $3 \mathrm{G}$ in a panel of 26 GICs are shown in the heat map. (B) Gene expression analysis of APOBEC3G in TCGA samples are shown in the heat map. CD44 is a marker of the mesenchymal subtype, whereas Olig-2 is the non-mesenchymal marker. (C) Expression of APOBEC3G was correlated with CD44 in the TCGA data. (D) The expression of APOBEC3G, CD44 and Olig2 in GICs (GSC268, GSC23, GSC231, GSC28, GSC20) and in GBM cell lines (A172, U343) was detected by Western blot analysis. Actin was used as the control. (E) TCGA data showed that patients with high expression of APOBEC3G had short survival durations. 
of APOBEC3G expression significantly inhibited cell proliferation in A172 (Figure 2B), U343 (Figure 2C), and GSC20 cells (Figure 2D) in comparison to scramble shRNA (SCR) transfected cells.

\section{APOBEC3G knockdown attenuates invasion of mesenchymal GICs and GBM cells}

Recent data suggest that $\mathrm{APOBEC} 3 \mathrm{G}$ can promote liver metastasis in colorectal cancer [19], but its role in GBM is not clear. To explore the role of APOBEC3G in the migration of GBM cell lines, we performed a woundhealing assay in A172 and U343 cells. Knockdown of APOBEC $3 \mathrm{G}$ impaired the migration of A172 cells by $36.3 \%(P<0.01)$ (Figure 3A) and U343 cells by $32.4 \%$ $(P<0.05)$ (Figure 3B) in comparison to scramble control cells after $18 \mathrm{~h}$ of observation. We further studied the invasion ability of glioma cells and performed matrigel transwell migration assays to demonstrate that knockdown of APOBEC $3 \mathrm{G}$ decreased the number of cells that migrated to the other side of transwell by $50.8 \%$ in A172 cells $(P<0.01)$ (Figure 3C) and $49.1 \%$ in U343 cells $(P<0.01)$ (Figure 3D). These results clearly show that targeting APOBEC3G attenuated the migration and invasive capabilities of GBM.

\section{Targeting APOBEC3G attenuates TGF $\beta$ signaling pathway}

To delineate the molecular mechanisms through which APOBEC3G regulates $\mathrm{CD}^{4} 4^{+}$glioma cell proliferation and migration, we investigated intracellular signaling pathways in APOBEC3G knockdown cells. TGF $\beta$ expression in malignant brain tumors was found to render the tumor cells survival advantage by enhancing cell growth, migration, invasion, angiogenesis, and immune suppression and stem cell properties. We analyzed the C-Bio database and data analysis showed expression of APOBEC3G correlated with the expression of TGF $\beta$ R 1 (Spearman's correlation: 0.36) (Figure 4A) and TGF $\beta 1$ (Spearman's correlation: 0.48) (Figure 4B). These preliminary data prompted us to further investigate the mechanism of APOBEC $3 \mathrm{G}$ focusing on TGF $\beta$ signaling pathway.

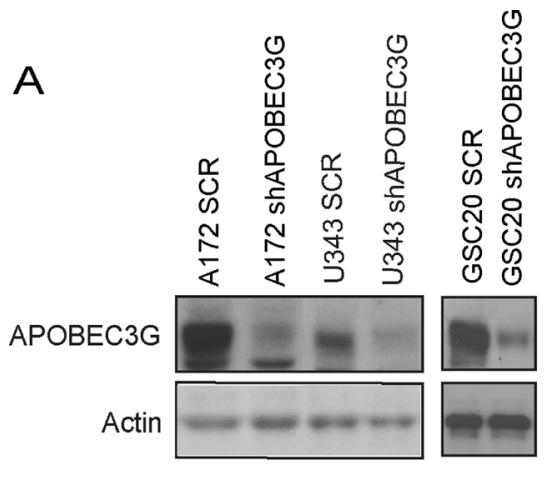

C

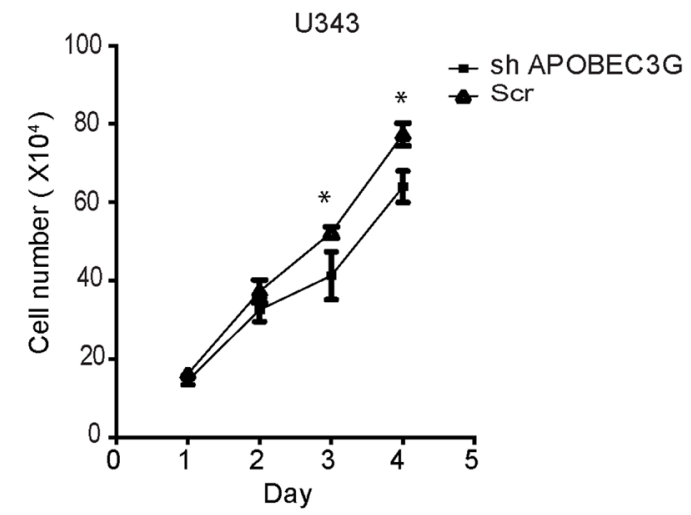

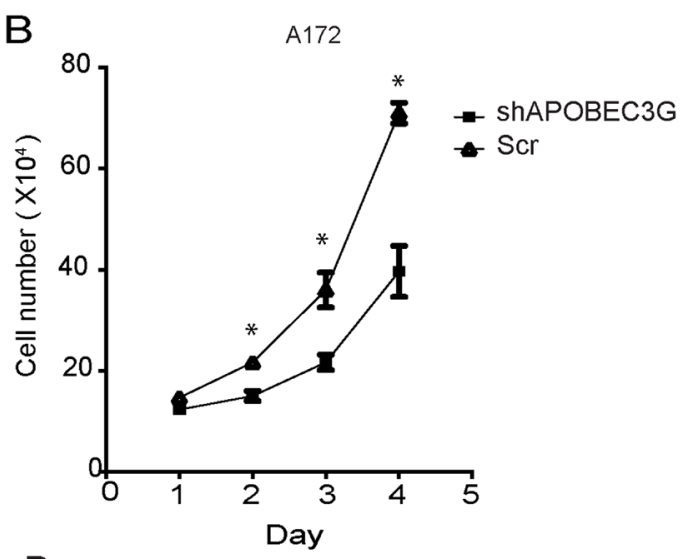

D

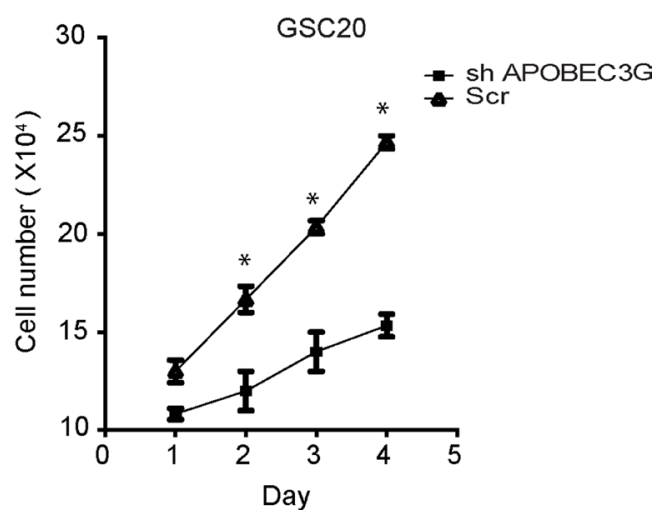

Figure 2: Depletion of APOBEC3G attenuated proliferation of mesenchymal GICs and GBM cells. (A) APOBEC3G was knocked down by lentivirus shRNAs in A172, U343 and GSC20, and the knockdown effect was confirmed by Western blot. Scramble sequence shRNA (SCR) was used as the control. (B-D) A172 (B), U343 (C) and GSC20 (D) APOBEC3G knock-down cells as well as SCR cells were seeded in 6 -well plates $\left(1 \times 10^{5}\right.$ cells/well $)$, cell numbers were counted and plotted every day for 4 days. Symbol $*$ means $p<0.01$. 
Canonically, TGF $\beta 1$ binds and activates TGF $\beta$ R, which phosphorylates Smad2, the key node of TGF $\beta$ signaling pathway. In A172 cells and GSC20 cells, TGF $\beta$ treatment induced Smad2 phosphorylation (Figure 4C and 4D), and as expected, the TGF $\beta$-induced Smad2 phosphorylation was abrogated by TGF $\beta R 1$ inhibitor LY2157299. Interestingly, knockdown of APOBEC3G by shRNA also markedly blocked TGF $\beta$ induced Smad2 phosphorylation (Figure 4C and 4D), suggesting APOBEC $3 \mathrm{G}$ is involved in TGF $\beta$ signaling pathway.

To further understand the role of APOBEC $3 \mathrm{G}$ in Smad2 deactivation in gliomas, we determined its role in mediating the expression of the Smad2-targeted genes
Thrombospondin 1 (TSP-1), matrix metallopeptidase 2 (MMP2) and TIMP metallopeptidase inhibitor 1 (TIMP-1). RT-PCRs were performed to compare the expression levels of Smad2-targeted genes between scramble and APOBEC3G shRNA transfected A172 cells. The results show that the expression of MMP2 and TSP-1 which play important role in cell migration, was decreased in shAPOBEC $3 \mathrm{G}$ cells compared to scramble controls (Figure 4E, left and middle panels); in contrast, expression of TIMP-1, an natural inhibitor of the matrix metalloproteinases was upregulated by APOBEC3G depletion (Figure 4E, right panel). Taken together, these results suggested that APOBEC $3 \mathrm{G}$ interferes with TGF $\beta$ signaling by affecting Smad2 phosphorylation and Smad2

\section{A}
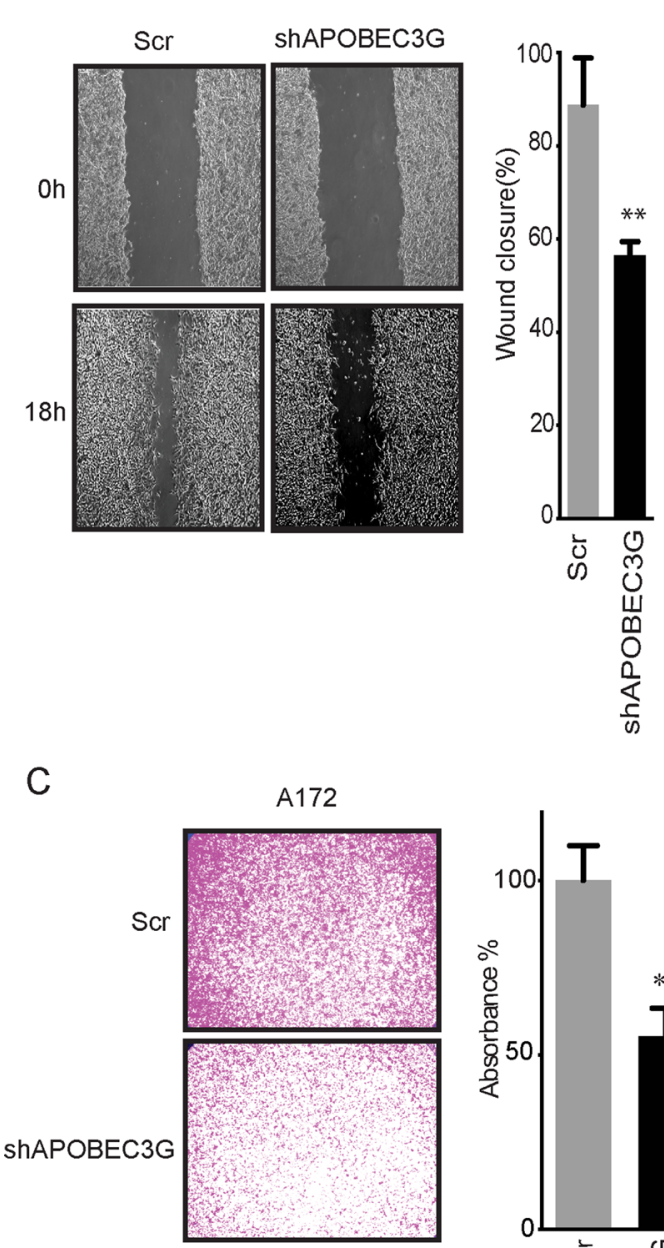

B
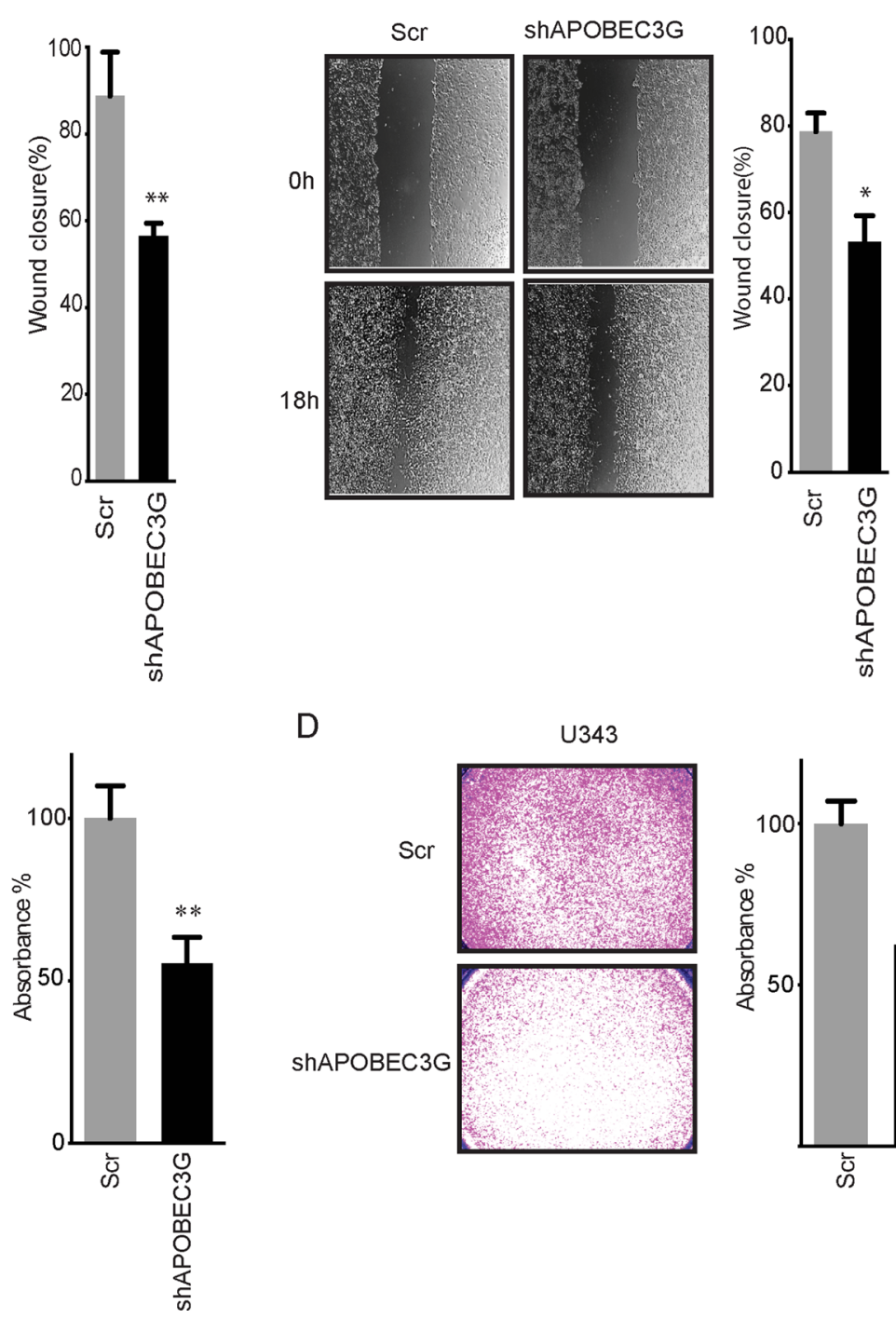

$\mathrm{D}$

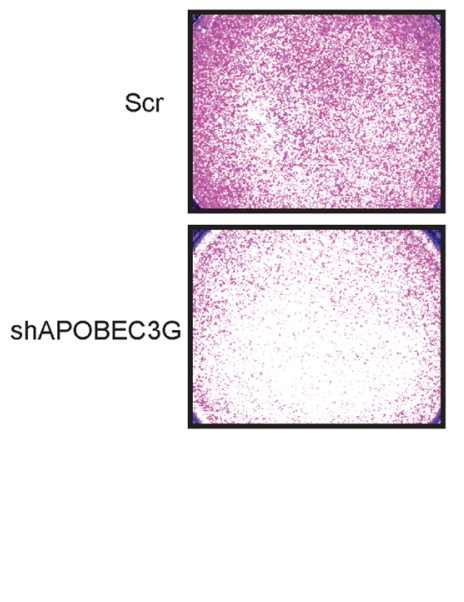

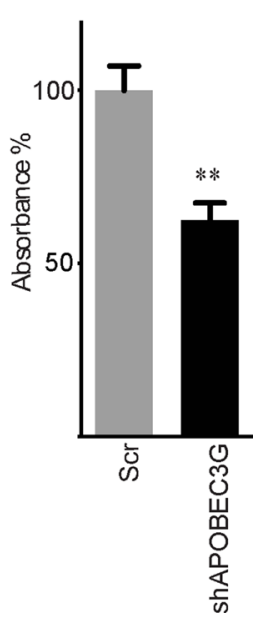

Figure 3: Targeting APOBEC3G impaired migration ability of mesenchymal GBM cell lines. (A-B) Micrographs showing wound healing assay of A172 (A) and U343 (B) APOBEC3G-depleted cells and SCR cells. The width of scratch at $0 \mathrm{~h}$ was considered to be $100 \%$. The relative migration distance of cells was demonstrated. (C-D) Micrographs showing matrigel transwell migration assay in A172 (C) and U343 (D) APOBEC3G-depleted cells and SCR cells. The cells migrated to the lower surface were quantified on the basis of the absorbance measured at $595 \mathrm{~nm}$. The absorbance of transwell of SCR cells was considered to be $100 \%$. Symbols $*$ and $* *$ mean $P<$ 0.05 and $p<0.01$, respectively. 
downstream target gene expression, which may account for APOBEC3G mediated proliferation and migration in mesenchymal GICs and GBM Cells.

\section{APOBEC3G knockdown sensitizes mesenchymal GICs and GBM cells to IR}

Recent reports have shown that APOBEC3G enhances lymphoma cell radioresistance by promoting DNA repair [27]. However, whether APOBEC3G influences the radioresistance of GBM cell lines is unknown. Therefore, we determined the effect of IR on A172-scramble cells and A172 APOBEC3G-shRNA cells. Cells were treated with or without IR (2 Gy) and allowed to recover and grow for 10 days. Treatment with 2 Gy of IR impaired A172 colony formation, but a significant number of cells survived (Figure 5A and 5B). Furthermore, targeting APOBEC3G sensitized A172 cells to radiation, as the combination of shAPOBEC $3 \mathrm{G}$ and IR significantly attenuated colony formation

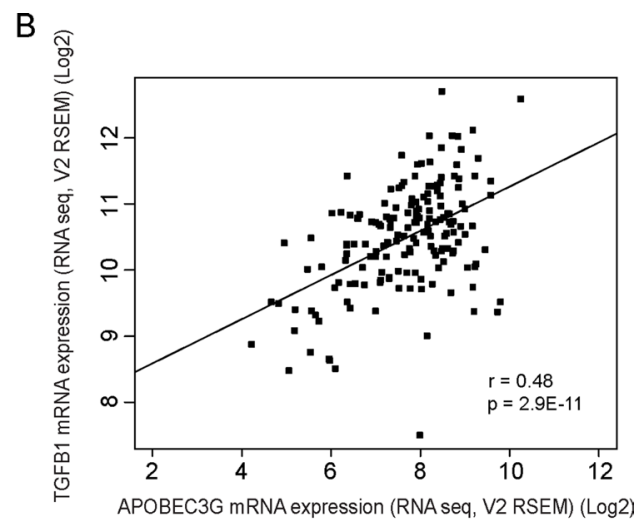

C
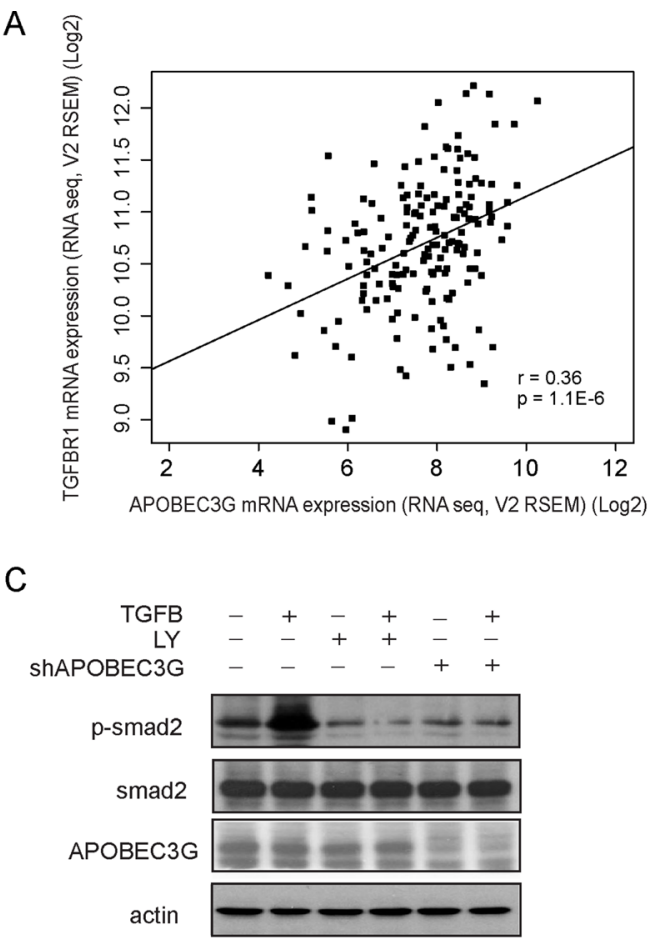

p-smad2/smad2 $\quad 1.00 \quad 2.32 \quad 0.39 \quad 0.190 .23 \quad 0.19$

A172

D

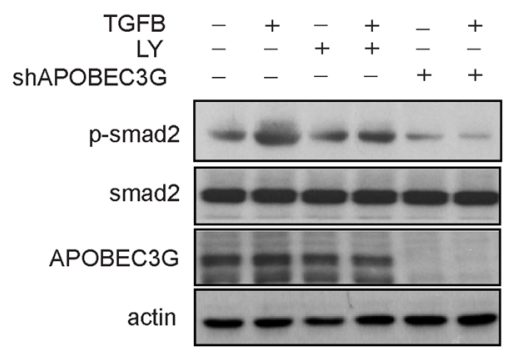

p-smad2/smad2 $1.002 .22 \quad 1.12 \quad 1.39 \quad 0.590 .43$

GSC20
$E$

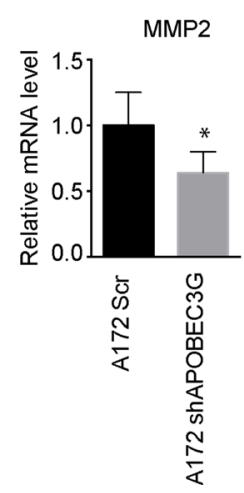

TSP-1

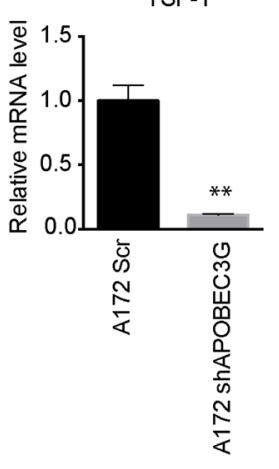

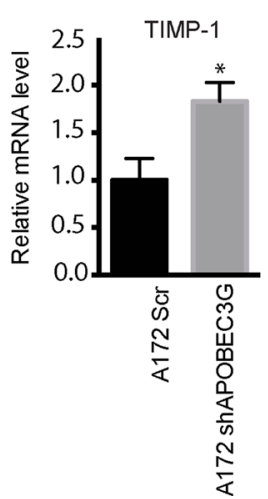

Figure 4: Depletion of APOBEC3G attenuated the TGF $\beta$ signaling pathway. (A-B) TCGA data showed that expression of TGFßR1 (A) and TGFß1(B) was correlated with APOBEC3G. (C-D) Immunoblotting analysis showed that shRNA targeting APOBEC3G reduced the expression of phosphorylated smad2. A172 cells (C) and GSC20 (D) transfected with scramble or APOBEC3G shRNA, treated with $2.5 \mathrm{ng} / \mathrm{ml}$ TGFß1, $10 \mathrm{mM}$ LY2157299 (TGFßR1 inhibitor), or combination for $24 \mathrm{~h}$, and cell lysis was analyzed by western blot with specific antibodies against phosphorylated smad2, total smad2 and APOBEC3G. The expression of p-smad2 and total smad 2 was quantified by Image-J and their ratio was shown in the bottom. (E) The expression of smad2 downstream target genes MMP2, TSP1 and TIMP-1 were analyzed in A172 cells transfected with scramble shRNA (A172SCR) or A712 APOBEC3G knock-down cells (A172 shAPOBEC3G) by quantitative-PCR. Symbols * and ** mean $P<0.05$ and $p<0.01$, respectively. 
(Figure 5A and 5B). In a limiting dilution colony formation assay, the cells were diluted and plated in each well (1-3 cells per well) of 96-well plates. Without IR, the sphere formation ability of scramble cells is similar to that of APOBEC3G knockdown cells. After 2 Gy IR, the colony formation of APOBEC3G knockdown cells was impaired by $35.1 \%(P<0.05)$ compared to that of scramble cells (Figure 5C). These data suggest that targeting APOBEC3G renders mesenchymal GICs and GBM cells sensitive to IR-induced cell death.

\section{APOBEC3G knockdown attenuates checkpoint activation of DNA repair pathway}

We next investigated the mechanism of radiosensitization in APOBEC3G knockdown cells. IR mainly causes cellular toxicity through the induction of DSBs that activate the DNA damage checkpoint signaling pathway [29, 31]. Checkpoint pathway activation initiates cell-cycle arrest with attempted DNA repair [32, 33]. Chk2 is the key checkpoint that promotes DNA repair after IR damage. As shown in Figure 5D and 5E, the expression of phosphorylated Chk2 was induced at 3 hours post 2Gy irradiation in SCR cells suggesting DNA repair is initiated. pChk2 expression was much lower in APOBEC3G knockdown cells compare with that in SCR cells at both 3 hours and 12 hours post IR, suggesting APOBEC3G depletion inhibited DNA repair. Consistently, we observed cleaved PARP, a marker for apoptosis, was induced in APOBE3G knockdown cells but not in SCR cells at $12 \mathrm{~h}$ post IR, demonstrating inhibition 2 Gy IR induced apoptosis in APOBE3G knocked-down cells but not in SCR cells. These data suggest that knockdown of APOBEC3G impairs activation of the DNA repair pathway and sensitizes irradiation induced- apoptosis in mesenchymal GICs and GBM cells.
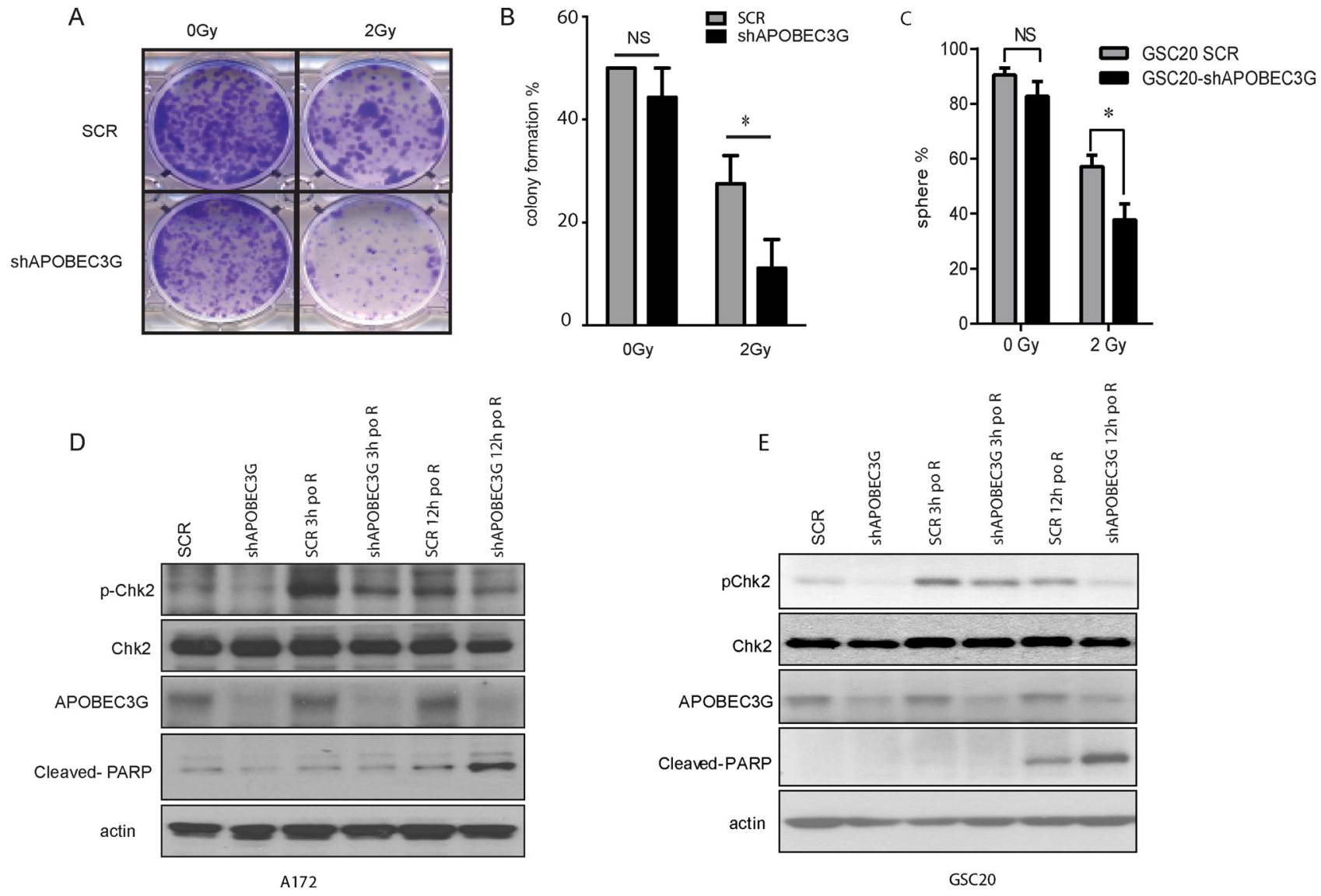

Figure 5: Inhibition of APOBEC3G increased the radiosensitivity of GBM cells and mesenchymal GICs. (A-B) Colony formation assay showed that APOBEC3G knockdown reduced the colony formation of A172 cells after IR (2 Gy). A172-SCR and A173shAPOBEC $3 \mathrm{G}$ were treated with or without IR ( $2 \mathrm{~Gy}$ ) before being allowed to recover and grow for 10 days. Representative pictures are illustrated (A) and colony number was counted from triplicate experiments (B). (C) Limiting dilution sphere formation assay demonstrated that targeting APOBEC $3 \mathrm{G}$ reduced sphere formation in GSC20 cells after IR (2 Gy). GSC20 cells were targeted with scramble or APOBEC3G shRNA, treated without or with IR (2 Gy), and diluted; 1-3 cells were plated and allowed to recover and grow for 21 days. Sphere-positive wells were counted, and the percentage of positive wells was calculated. (D-E) Immunoblot analysis showed that APOBEC3G knockdown impaired the DNA repair response after IR. A172 cells (D) and GSC20 (E) with scramble or APOBEC3G shRNA were exposed to 2 Gy IR. Cells were harvested before IR or $3 \mathrm{~h}$ or $12 \mathrm{~h}$ after IR for an immunoblot analysis with specific antibodies against phosphorylated Chk2, total Chk2, Cleaved-PARP, and APOBEC3G. 


\section{DISCUSSION}

GBMs of the mesenchymal subclass have been linked with high aggressiveness and resistance to treatment, whereas patients with a proneural signature were reported to perform better in the clinic with respect to survival and treatment responses $[6,34,35]$. Up to date, many small molecule inhibitors targeting specific pathway may yield stronger specificity and thus improve killing tumor effects. Mesenchymal identity is the hallmark of GBM cell aggressiveness with tight correlation to the poor outcome of patients. In our analysis of GBM patient derived GICs, we report that in mesenchymal GICs, genes involved in TGF $\beta$ signaling pathways including APOBEC $3 \mathrm{G}$, is significantly up-regulated compared with non-mesenchymal GICs. Among APOBEC3 families, APOBE3G expression is the most significant unregulated target $(1.8$ folds and FDR $=0.0018)$, APOBEC3C and APOBEC $3 \mathrm{~F}$ showed moderate or slight upregulation (1.4 folds for APOBEC3C and 1.1 folds for APOBEC3F) (Supplementary Figure 1). In our studies, inhibition of APOBEC3G by specific shRNA attenuates invasion, migration and in vitro growth. APOBEC $3 \mathrm{G}$ is highly expressed in clinical GBM tissues and mesenchymal GICs display a significantly higher radio resistance, with markedly elevated levels of expression of genes associated with DNA repair. Inhibition of APOBEC3G reverses the radiation-resistant phenotype of mesenchymal GICs.

A recent study demonstrated that $\mathrm{APOBEC} 3 \mathrm{G}$ down regulates miR-29 expression and hampers miR-29 activity in repressing MMP2, which promotes hepatic metastasis of colorectal cancer [19]. Whether APOBEC3G can promote GBM cell survival and invasion is unknown. Our data show that knockdown of APOBEC3G attenuates the proliferation and invasion of GBM cell lines. We show that targeting APOBEC3G can decrease activation of the TGF $\beta$ signaling pathway, which may account for the effects of APOBEC $3 \mathrm{G}$ on GBM. We show that targeting APOBEC $3 \mathrm{G}$ affected TGF $\beta$-induced smad2 activation bridging APOBEC $3 \mathrm{G}$ and the TGF $\beta$ pathway and illustrate the functions and mechanisms of APOBEC $3 \mathrm{G}$ in GBM. Concomitant with smad2 deactivation, we show decrease in TSP-1, MMP2, increase of TIMP-1, all are smad2 targeted genes clearly showing that targeting APOBEC 3G regulates TGF $\beta 1$ signaling pathway. However, how APOBEC $3 \mathrm{G}$ regulates the TGF $\beta$ pathway remains unknown. As a cytidine deaminase, APOBEC3G deaminates cytidine residues and causes guanosineto-adenosine hypermutation. In addition, it suppresses translation by inhibiting the translocation of reverse transcriptase along template RNA [16] or binding to 3'UTR of target mRNA [36]. More importantly, Sharma et al. recently have discovered that APOBEC3G causes sitespecific C-to-U editing of mRNAs from over 600 genes $[37,38]$. Therefore, future studies focused on whether the regulation of TGF $\beta$ pathway by $\mathrm{APOBEC} 3 \mathrm{G}$ is dependent on its ssDNA editing, elongation blockage or RNA editing function will be important to understand its mechanism and to determinate its clinical application in GBM.

Ionizing radiation is one of the most effective therapies for GBM, but radiotherapy is only palliative because of radioresistance [39]. Checkpoint pathways have cytoprotective roles that allow cells to survive after DNA repair [31]. Thus, DNA damage checkpoint responses play an important role in cellular radiosensitivity [33, 40-42]. Chk2 represents a conserved signaling component that responds to DNA damage and protects genomic integrity [43]. Activation of the Chk2 kinase in response to DNA damage is a multistep exquisite process $[28,44]$ and Chk2 activation can promote DNA repair after IR-induced DNA damage to render cancer cells more resistant to radiation therapy.

Several types of cancer cells, such as lymphoma and myeloma cells, display efficient repair of genomic DSBs induced by ionizing radiation and promote cell survival after IR. Interestingly, these cells express higher levels of APOBEC3G than do their paired normal cells [45]. In the current study, we identified a new function of APOBEC $3 \mathrm{G}$ in promoting the checkpoint activation and radioresistance of GBM cell lines by regulating the key molecular checkpoint, Chk2, in cellular responses to DNA damage. Notably, depletion of APOBEC3G alone did not induce apoptosis but potentiated irradiation-induced apoptosis, suggesting that APOBEC $3 \mathrm{G}$ plays an important role in DNA repair for irradiation-induced DNA damage and contributes to the radioresistance.

In summary, our data showed that APOBEC3G is preferentially expressed in mesenchymal gliomas and affects Smad2 activation, and enhances checkpoint activation. APOBEC3G mediated checkpoint activation through Chk2 is one of the critical regulatory mechanisms that underlies the preferential DNA damage checkpoint response and radioresistance of GICs. Thus, antiAPOBEC $3 G$ therapy may synergize with radiotherapy and other current treatments to overcome the therapeutic resistance of gliomas. APOBEC $3 \mathrm{G}$ represents a potential molecular target for novel therapeutics that will improve the treatment outcome of glioma patients.

\section{MATERIALS AND METHODS}

\section{Cell lines and reagents}

Glioma initiating Cells (GICs) were established by isolating neurosphere-forming cells from surgical specimens of human GBM, as described previously [9]. These GICs were cultured as GBM neurospheres in DMEM/F12 medium containing B27 supplement (Invitrogen, Carlsbad, CA) and basic fibroblast growth factor and epidermal growth factor $(20 \mathrm{ng} / \mathrm{ml}$ each). This study was approved by the institutional review board of The University of Texas MD Anderson Cancer Center 
(Houston, TX, USA). Both unsupervised and supervised approaches were used to classify GICs into molecular subtypes [5]. Cells were authenticated by testing short tandem repeats (STR) using the Applied Biosystems AmpFISTR Identifier kit (Foster City, CA). The last authentication testing was done in March 2014. Glioma cell lines were grown in DMEM supplemented with $10 \%$ FBS. The TGFßR1 kinase inhibitor (LY 2157299) was purchased from Selleck Chemicals (Houston, TX) and was dissolved in dimethyl sulfoxide (Sigma-Aldrich, St. Louis, MO, USA) to a concentration of $10 \mathrm{mmol} / \mathrm{L}$. Recombinant human TGF $\beta 1$ was purchased from R\&D Systems (Minneapolis, MN, USA), reconstituted in 20 $\mu \mathrm{g} / \mathrm{mL}$ in sterile $4 \mathrm{mM} \mathrm{HCl}$ containing $1 \mathrm{mg} / \mathrm{mL}$ bovine serum albumin and stored at $-20^{\circ} \mathrm{C}$ and further diluted to an appropriate final concentration in DMEM/F12 medium at the time of use.

\section{Western blot analysis}

Cells were harvested in lysis solution, as previously described [46], and subjected to Western blotting analysis. Membranes were probed with the following primary antibodies: anti-phosphorylated smad2, anti-total smad2, anti-TGFßR1, anti-phosphorylated Chk2, anti-total Chk2, anti-PARP, anti-CD44, anti-Olig2 (all from Cell Signaling, Boston, MA, USA), and anti-APOBEC3G (Proteintech, Chicago, IL, USA). Anti- $\beta$-actin antibody was purchased from Sigma (St. Louis, MO, USA) and used as the loading control.

\section{Cell growth assay}

GICs and GBM cell lines were seeded in 6-well plates $\left(1 \times 10^{5}\right.$ cells/well $)$. Cell numbers were counted every day for 4 days using a hemocytometer. The experiment was repeated three times, and the average value was reported.

\section{Wound healing assay}

Confluent A172 and U343 cells were transfected with scramble shRNA or APOBEC3G shRNA. At $48 \mathrm{~h}$ after transfection, a cell scratch spatula was used to make a scratch in the cell monolayer. Pictures of the scratches were taken ( $\times 5$ magnification) using a digital camera system coupled with a microscope was taken after $18 \mathrm{~h}$. The software program Image $\mathrm{J}$ was used to determine the migration distance (in pixels).

\section{Matrigel transwell migration assay}

Cell migration assays were performed on polycarbonate membrane inserts $(8-\mu \mathrm{m}$ pore size; Greiner Bio-One, Inc., Longwood, FL, USA). A172 and U343 cells were washed with PBS and serum-free medium prior to being resuspended in fresh serum-free medium. 50,000 cells in $250 \mu \mathrm{l}$ of serum-free medium were plated over the inner chamber of Matrigel-coated inserts in a 24-well tissue culture plate and $500 \mu \mathrm{l}$ of $3 \%$ fetal bovine serum medium was placed in the outer chamber of the insert. Plates were incubated at $37^{\circ} \mathrm{C}$ for $24 \mathrm{~h}$. The cells that had migrated through to the lower surface of the ECM layer were stained with $1 \%$ crystal violet and dissolved in $2 \%$ deoxycholic acid. Cells were quantified on the basis of the absorbance, measured at $595 \mathrm{~nm}$. Each experiment was performed in triplicate.

\section{Knockdown of APOBEC3G by lentiviral shRNA}

The lentiviral vectors pGIPZ-mediated shRNA for APOBEC3G (clone ID: V3LHS_303306) and scramble was purchased from GE Healthcare Dharmacon (Pittsburgh, PA, USA), and transfected into 293FT cells according to the manufacturer's instructions. In brief, lentiviral particles expressing targeting or control scramble shRNA were produced in HEK293FT cells with a mixed set of packing plasmids and the viruses were concentrated and titered as previously described [47]. The produced lentiviruses were concentrated using the Centricon Plus-20 centrifugal filter device (Millipore, Billerica, MA, USA). The lentiviral stock was titered and stored at $-80^{\circ} \mathrm{C}$. GSC20, A172, or U343 cells were infected with lentivirus for 24 hours. Cells were washed and cultured with regular complete medium for 2 days in the presence of $2.5 \mu \mathrm{g} / \mathrm{ml}$ puromycin. Finally, the cells were washed and analyzed for protein expression by Western blotting.

\section{Colony formation assay}

Cells were treated with IR (2 Gy) and plated in 6-well plates (2000 cells per well), cultured in DMEM/F12 medium with $10 \%$ FBS for 10 days. Cells were stained with $1 \%$ crystal violet and dissolved in $2 \%$ deoxycholic acid. Each experiment was performed in triplicate.

\section{Limiting dilution sphere formation assay}

Cells were treated with IR (2 Gy), and accutased to generate single-cells suspension, diluted serially to plate 1-3 cells per well in 96-well plates with 3 plates for each condition per cell line, cultured for 3 weeks DMEM/F12 medium supplemented with B27, EGF and FGF. The number of wells with neuro-sphere was counted and the percentage of positive wells was calculated. Each experiment was performed in triplicate.

\section{RNA extraction and real-time quantitative polymerase chain reaction}

Total RNA was extracted from A172 cells using the RNeasy Mini kit (Qiagen, Valencia, CA, USA), according 
to the manufacturer's instructions. Real-time quantitative PCR was performed with the SuperScript III One-Step RT-PCR System and sybgreen DNA polymerase (Invitrogen, GrandIsland, NY, USA), according to the manufacturer's instructions. Primers for quantitative PCR are listed in Supplementary Table 1.

\section{Author contributions}

D Koul, W Yung and S Wu designed the study. Y Wang, S Wu, S Wang and R Ezhilarasan, A wali conducted the experiment and collected the data. Y Wang and $\mathrm{S} \mathrm{Wu}$ did the statistical analysis and prepared the manuscript. S Zheng did the bioinformatics study and interpretation. D Koul, E Sulman and W Yung revised the manuscript. All authors read and approved the final manuscript.

\section{CONFLICTS OF INTEREST}

The authors (W. K. Alfred Yung) disclose conflict of interest as a consultant with DNATrix. All other authors have no conflict of interest to disclose.

\section{FUNDING}

This study was funded by a CPRIT grant (RP120256 to W. K. A. Yung), National Brain Tumor Society (Defeat GBM) Grant, National Foundation for Cancer Research (NFCR) to W. K. A. Yung, a SPORE grant (P50 CA127001 to F. F. Lang), and a Cancer Center Support Grant (CA016672 to Ronald A. DePinho).

\section{REFERENCES}

1. Wen PY, Kesari S. Malignant gliomas in adults. N Engl J Med. 2008; 359:492-507.

2. Stupp R, Mason WP, van den Bent MJ, Weller M, Fisher B, Taphoorn MJ, Belanger K, Brandes AA, Marosi C, Bogdahn U, Curschmann J, Janzer RC, Ludwin SK, et al, and European Organisation for Research and Treatment of Cancer Brain Tumor and Radiotherapy Groups, and National Cancer Institute of Canada Clinical Trials Group. Radiotherapy plus concomitant and adjuvant temozolomide for glioblastoma. N Engl J Med. 2005; 352:987-96.

3. Stupp R, Hegi ME, Mason WP, van den Bent MJ, Taphoorn MJ, Janzer RC, Ludwin SK, Allgeier A, Fisher B, Belanger K, Hau P, Brandes AA, Gijtenbeek J, et al, and European Organisation for Research and Treatment of Cancer Brain Tumour and Radiation Oncology Groups, and National Cancer Institute of Canada Clinical Trials Group. Effects of radiotherapy with concomitant and adjuvant temozolomide versus radiotherapy alone on survival in glioblastoma in a randomised phase III study: 5-year analysis of the EORTC-NCIC trial. Lancet Oncol. 2009; 10:459-66.
4. Cloughesy TF, Cavenee WK, Mischel PS. Glioblastoma: from molecular pathology to targeted treatment. Annu Rev Pathol. 2014; 9:1-25.

5. Verhaak RG, Hoadley KA, Purdom E, Wang V, Qi Y, Wilkerson MD, Miller CR, Ding L, Golub T, Mesirov JP, Alexe G, Lawrence M, O'Kelly M, et al, and Cancer Genome Atlas Research Network. Integrated genomic analysis identifies clinically relevant subtypes of glioblastoma characterized by abnormalities in PDGFRA, IDH1, EGFR, and NF1. Cancer Cell. 2010; 17:98-110.

6. Phillips HS, Kharbanda S, Chen R, Forrest WF, Soriano RH, Wu TD, Misra A, Nigro JM, Colman H, Soroceanu L, Williams PM, Modrusan Z, Feuerstein BG, Aldape K. Molecular subclasses of high-grade glioma predict prognosis, delineate a pattern of disease progression, and resemble stages in neurogenesis. Cancer Cell. 2006; 9:157-73.

7. Huse JT, Phillips HS, Brennan CW. Molecular subclassification of diffuse gliomas: seeing order in the chaos. Glia. 2011; 59:1190-99.

8. Carro MS, Lim WK, Alvarez MJ, Bollo RJ, Zhao X, Snyder EY, Sulman EP, Anne SL, Doetsch F, Colman H, Lasorella A, Aldape K, Califano A, Iavarone A. The transcriptional network for mesenchymal transformation of brain tumours. Nature. 2010; 463:318-25.

9. Bhat KP, Salazar KL, Balasubramaniyan V, Wani K, Heathcock L, Hollingsworth F, James JD, Gumin J, Diefes KL, Kim SH, Turski A, Azodi Y, Yang Y, et al. The transcriptional coactivator TAZ regulates mesenchymal differentiation in malignant glioma. Genes Dev. 2011; 25:2594-609.

10. Salter JD, Bennett RP, Smith HC. The APOBEC Protein Family: United by Structure, Divergent in Function. Trends Biochem Sci. 2016; 41:578-94.

11. Sheehy AM, Gaddis NC, Choi JD, Malim MH. Isolation of a human gene that inhibits HIV-1 infection and is suppressed by the viral Vif protein. Nature. 2002; 418:646-50.

12. Jarmuz A, Chester A, Bayliss J, Gisbourne J, Dunham I, Scott J, Navaratnam N. An anthropoid-specific locus of orphan C to U RNA-editing enzymes on chromosome 22. Genomics. 2002; 79:285-96.

13. Harris RS, Bishop KN, Sheehy AM, Craig HM, PetersenMahrt SK, Watt IN, Neuberger MS, Malim MH. DNA deamination mediates innate immunity to retroviral infection. Cell. 2003; 113:803-09.

14. Lecossier D, Bouchonnet F, Clavel F, Hance AJ. Hypermutation of HIV-1 DNA in the absence of the Vif protein. Science. 2003; 300:1112.

15. Norman JM, Mashiba M, McNamara LA, Onafuwa-Nuga A, Chiari-Fort E, Shen W, Collins KL. The antiviral factor APOBEC3G enhances the recognition of HIV-infected primary T cells by natural killer cells. Nat Immunol. 2011; 12:975-83.

16. Bishop KN, Verma M, Kim EY, Wolinsky SM, Malim MH. APOBEC3G inhibits elongation of HIV-1 reverse transcripts. PLoS Pathog. 2008; 4:e1000231. 
17. Sharma S, Garg A, Dhanda RS, Kaul D. APOBEC3G governs the generation of truncated AATF protein to ensure oncogenic transformation. Cell Biol Int. 2016; 40:1366-71.

18. Lan H, Jin K, Gan M, Wen S, Bi T, Zhou S, Zhu N, Teng L, Yu W. APOBEC3G expression is correlated with poor prognosis in colon carcinoma patients with hepatic metastasis. Int J Clin Exp Med. 2014; 7:665-72.

19. Ding Q, Chang CJ, Xie X, Xia W, Yang JY, Wang SC, Wang Y, Xia J, Chen L, Cai C, Li H, Yen CJ, Kuo HP, et al. APOBEC3G promotes liver metastasis in an orthotopic mouse model of colorectal cancer and predicts human hepatic metastasis. J Clin Invest. 2011; 121:4526-36.

20. Burns MB, Lackey L, Carpenter MA, Rathore A, Land AM, Leonard B, Refsland EW, Kotandeniya D, Tretyakova N, Nikas JB, Yee D, Temiz NA, Donohue DE, et al. APOBEC3B is an enzymatic source of mutation in breast cancer. Nature. 2013; 494:366-70.

21. Bennett RP, Presnyak V, Wedekind JE, Smith HC. Nuclear Exclusion of the HIV-1 host defense factor APOBEC3G requires a novel cytoplasmic retention signal and is not dependent on RNA binding. J Biol Chem. 2008; 283:7320-27.

22. Rich JN. The role of transforming growth factor-beta in primary brain tumors. Front Biosci. 2003; 8:e245-60.

23. Platten M, Wick W, Weller M. Malignant glioma biology: role for TGF-beta in growth, motility, angiogenesis, and immune escape. Microsc Res Tech. 2001; 52:401-10.

24. Mani SA, Guo W, Liao MJ, Eaton EN, Ayyanan A, Zhou AY, Brooks M, Reinhard F, Zhang CC, Shipitsin M, Campbell LL, Polyak K, Brisken C, et al. The epithelialmesenchymal transition generates cells with properties of stem cells. Cell. 2008; 133:704-15.

25. Shuang ZY, Wu WC, Xu J, Lin G, Liu YC, Lao XM, Zheng L, Li S. Transforming growth factor- $\beta 1$-induced epithelial-mesenchymal transition generates ALDH-positive cells with stem cell properties in cholangiocarcinoma. Cancer Lett. 2014; 354:320-28.

26. Joseph JV, Conroy S, Tomar T, Eggens-Meijer E, Bhat K, Copray S, Walenkamp AM, Boddeke E, Balasubramanyian V, Wagemakers $M$, den Dunnen WF, Kruyt FA. TGF- $\beta$ is an inducer of ZEB1-dependent mesenchymal transdifferentiation in glioblastoma that is associated with tumor invasion. Cell Death Dis. 2014; 5:e1443.

27. Nowarski R, Wilner OI, Cheshin O, Shahar OD, Kenig E, Baraz L, Britan-Rosich E, Nagler A, Harris RS, Goldberg M, Willner I, Kotler M. APOBEC3G enhances lymphoma cell radioresistance by promoting cytidine deaminase-dependent DNA repair. Blood. 2012; 120:366-75.

28. Bartek J, Lukas J. Chk1 and Chk2 kinases in checkpoint control and cancer. Cancer Cell. 2003; 3:421-29.

29. Lukas J, Lukas C, Bartek J. Mammalian cell cycle checkpoints: signalling pathways and their organization in space and time. DNA Repair (Amst). 2004; 3:997-1007.
30. Tusher VG, Tibshirani R, Chu G. Significance analysis of microarrays applied to the ionizing radiation response. Proc Natl Acad Sci USA. 2001; 98:5116-21.

31. Harper JW, Elledge SJ. The DNA damage response: ten years after. Mol Cell. 2007; 28:739-45.

32. Reinhardt HC, Yaffe MB. Kinases that control the cell cycle in response to DNA damage: Chk1, Chk2, and MK2. Curr Opin Cell Biol. 2009; 21:245-55.

33. Kastan MB, Bartek J. Cell-cycle checkpoints and cancer. Nature. 2004; 432:316-23.

34. Mao P, Joshi K, Li J, Kim SH, Li P, Santana-Santos L, Luthra S, Chandran UR, Benos PV, Smith L, Wang M, Hu B, Cheng SY, et al. Mesenchymal glioma stem cells are maintained by activated glycolytic metabolism involving aldehyde dehydrogenase 1A3. Proc Natl Acad Sci USA. 2013; 110:8644-9.

35. Pelloski CE, Mahajan A, Maor M, Chang EL, Woo S, Gilbert M, Colman H, Yang H, Ledoux A, Blair H, Passe S, Jenkins RB, Aldape KD. YKL-40 expression is associated with poorer response to radiation and shorter overall survival in glioblastoma. Clin Cancer Res. 2005; 11:3326-34.

36. Garg A, Kaul D, Chauhan N. APOBEC3G governs to ensure cellular oncogenic transformation. Blood Cells Mol Dis. 2015; 55:248-54.

37. Sharma S, Patnaik SK, Taggart RT, Kannisto ED, Enriquez SM, Gollnick P, Baysal BE. APOBEC3A cytidine deaminase induces RNA editing in monocytes and macrophages. Nat Commun. 2015; 6:6881.

38. Sharma S, Patnaik SK, Taggart RT, Baysal BE. The doubledomain cytidine deaminase APOBEC3G is a cellular sitespecific RNA editing enzyme. Sci Rep. 2016; 6:39100.

39. Garden AS, Maor MH, Yung WK, Bruner JM, Woo SY, Moser RP, Lee YY. Outcome and patterns of failure following limited-volume irradiation for malignant astrocytomas. Radiother Oncol. 1991; 20:99-110.

40. Sancar A, Lindsey-Boltz LA, Unsal-Kaçmaz K, Linn S. Molecular mechanisms of mammalian DNA repair and the DNA damage checkpoints. Annu Rev Biochem. 2004; 73:39-85.

41. Abraham RT. Cell cycle checkpoint signaling through the ATM and ATR kinases. Genes Dev. 2001; 15:2177-96.

42. Rieger KE, Chu G. Portrait of transcriptional responses to ultraviolet and ionizing radiation in human cells. Nucleic Acids Res. 2004; 32:4786-803.

43. Bartek J, Falck J, Lukas J. CHK2 kinase-a busy messenger. Nat Rev Mol Cell Biol. 2001; 2:877-86.

44. Ahn J, Urist M, Prives C. The Chk2 protein kinase. DNA Repair (Amst). 2004; 3:1039-47.

45. Nowarski R, Kotler M. APOBEC3 cytidine deaminases in double-strand DNA break repair and cancer promotion. Cancer Res. 2013; 73:3494-98. 
46. Koul D, Jasser SA, Lu Y, Davies MA, Shen R, Shi Y, Mills GB, Yung WK. Motif analysis of the tumor suppressor gene MMAC/PTEN identifies tyrosines critical for tumor suppression and lipid phosphatase activity. Oncogene. 2002; 21:2357-64.
47. Bao S, Wu Q, Li Z, Sathornsumetee S, Wang H, McLendon RE, Hjelmeland AB, Rich JN. Targeting cancer stem cells through L1CAM suppresses glioma growth. Cancer Res. 2008; 68:6043-48. 